\title{
LIPSCHITZ AND QUASICONFORMAL FLATTENING OF SPHERES AND CELLS
}

\author{
D. B. GAULD and J. VÄISÄLÄ
}

\section{Introduction}

1.1. We consider embeddings in three categories: TOP (continuous maps), LQC (locally quasiconformal maps) and LIP (locally Lipschitz maps). For compact objects, LQC maps are quasiconformal, and LIP maps are Lipschitz. Let $C$ be one of these categories, and let $X$ be a TOP $k$-sphere in the ordinary $n$-sphere $S^{n}$. We say that $X$ is locally $C$-flat if every point of $X$ has a neighbourhood $U$ such that $(U, U \cap X)$ is $C$-homeomorphic to $\left(R^{n}, R^{k}\right)$. It is well known that for $k \neq n-2$, local TOP flatness implies flatness in the sense that $\left(S^{n}, X\right)$ is homeomorphic to $\left(S^{n}, S^{k}\right)$, see [Ru, p. 48 and p. 158].

The main purpose of this paper is to consider similar questions in LQC and LIP. In particular, we prove in both categories that if $X$ is locally $C$-flat and if $4 \neq k \neq n-2$, then $\left(S^{n}, X\right)$ is $C$-homeomorphic to $\left(S^{n}, S^{k}\right)$. A related result on extending embeddings is also given, without the restriction $k \neq 4$. We also consider the analogous problem in which $X$ is a $k$-cell. Special cases have been considered in [Ti], [Ri], [LeV 2, p. 103], [Ge, p. 167], [NV], [LuV, 7.8], [GV], and [Ga].

Our main tool is a variation of engulfing, called encasing and considered in $\S 2$. The proofs of the main theorem in Sections 3 and 4 consist essentially of three steps. The first step involves the combining of the local collars given by the definition of local $C$-flatness. Using the encasing lemma of $\S 2$, we are able to combine sufficient collars so that $X$ is covered by just two of them.

The second step involves the enlargement of the two remaining collars so that their union is the whole of $S^{n}$. This involves an appeal to the known topological results together with PL approximation.

The third step combines these two enlarged collars to give us the required results. Here we make use of relative versions of the Schoenflies theorem, which are considered in 2.4 .

1.2. We shall several times make use of the fact that for $n \neq 4$, every homeomorphism $f: R^{n} \rightarrow R^{n}$ can be approximated by PL homeomorphisms. For $n=2$, this is proved in [Mo 2, p. 49], and for $n=3$, in [Mo 1]. For $n \geqq 5$, it follows from [Ru, Theorem 4.11.1, p. 194] and from the stable homeomorphism theorem [Ki, 
p. 575]. The latter result is very deep, but we could each time avoid it by using an engulfing argument instead. We shall not do it, however, because it would make the proofs longer and also make it necessary to give separate proofs for the cases $n \leqq 3$ and $n \geqq 5$.

1.3. Notation and terminology. The standard orthonormal basis of $R^{n}$ is written as $e_{1}, \ldots, e_{n}$, and we use the euclidean norm $|x|=\left(x_{1}^{2}+\ldots+x_{n}^{2}\right)^{1 / 2}$. If $k<n$, we identify $R^{k}$ with the subspace of $R^{n}$ spanned by $e_{1}, \ldots, e_{k}$. We let $\bar{R}^{n}=R^{n} \cup\{\infty\}$ denote the one-point compactification of $R^{n}$. The following notation is used for certain subsets of $R^{n}: R_{+}^{n}=\left\{x: x_{n} \geqq 0\right\}, B^{n}(r)=\{x:|x|<r\}, B_{+}^{n}(r)=B^{n}(r) \cap R_{+}^{n}$, $S^{n-1}(r)=\partial B^{n}(r), I^{n}(r)=[-r, r]^{n}, \Sigma^{n-1}(r)=\partial I^{n}(r)$. For $r=1$ we use the abbreviations $B^{n}, B_{+}^{n}, S^{n-1}, I^{n}, \Sigma^{n-1}$. Note that $B^{n}$ is the open unit ball.

The letter $C$ will always mean one of the categories TOP, LQC, LIP. However, our results are well known for $C=$ TOP. For the basic theory of LQC and LIP maps, we refer to [LeV 2], [Vä], and [LuV]. The objects of LQC are subsets $A$ of $\bar{R}^{n}$ such that $A \subset \mathrm{cl}$ int $A$. An embedding $f: A \rightarrow \bar{R}^{n}$ is LQC at $x \in A$ if $x$ has a neighbourhood $U$ such that $f$ is quasiconformal in $\operatorname{int}(U \cap A)$. By a one-dimensional quasiconformal map we mean a strictly monotone quasisymmetric function $[\mathrm{LeV} 2$, p. 88].

We shall use the spherical metric [Vä, p. 37] in $\bar{R}^{n}$. Then $\bar{R}^{n}, S^{n}$ and $\Sigma^{n}$ are mutually lipeomorphic, and we may freely replace one of them by another.

By an $n$-cell we mean a set homeomorphic to $I^{n}$.

1.4. Remark. When our manuscript was completed, the paper by Brakes $[\mathrm{Br}]$ appeared. It contains essentially the TOP case of the relative Schoenflies theorem 2.4 and a short proof of the TOP flatness of locally flat $k$-spheres in $S^{n}, k \leqq n-3$. We have incorporated his technique into the proofs of Theorems 3.3 and 3.4.

Acknowledgement. The first-named author wishes to express his thanks to the University of Auckland, from which he was on leave, and the University of Helsinki, his host during the period of this research.

\section{Encasing}

2.1. Definitions. A C-manifold, possibly with boundary, is defined in the standard way using atlases. For $C=\mathrm{LIP}$, an alternative definition is given in [LuV, 3.1].

Let $M$ be an $n$-dimensional $C$-manifold and let $X \subset M$ be a $k$-dimensional TOP submanifold. A set $A \subset X$ is $C$-encasable in $(M, X)$ if there is a $C$-embedding $h: B^{n} \rightarrow M$ such that $A \subset h B^{n}$ and $h^{-1} X$ is either $B^{k}$ or $B_{+}^{k}$. We also say that $h$ is a $C$-encasing of $A$ in $(M, X)$. If every point of $X$ is $C$-encasable in $(M, X), X$ is locally $C$-flat in $M$. 
With $M$ as in the previous paragraph, let $g: Y \rightarrow M$ be an embedding of the subset $Y$ of $\bar{R}^{k}$. Set $X=g Y$. A set $A \subset X$ is $(C, g)$-encasable if there is a neighbourhood $U$ of $g^{-1} A$ in $\bar{R}^{n}$ and a $C$-embedding $h: U \rightarrow M$ such that $(U, U \cap Y)$ is $C$-homeomorphic to $\left(B^{n}, B^{k}\right)$ or to $\left(B^{n}, B_{+}^{k}\right)$, that $h^{-1} X=U \cap Y$, and that $h=g$ in $U \cap Y$. Call the embedding $h$ a $(C, g)$-encasing of $A$. If every point of $X$ is $(C, g)$ encasable, say that $g$ is a locally $C$-flat embedding.

2.2. Lemma. Suppose that $A$ is a PL $k$-cell, $B \subset \partial A$ is a PL $(k-1)$-cell, $U$ is a neighbourhood of $B$ in $A$, and $C \subset A$ a compact set such that $C \cap \partial A \subset \operatorname{int} B$. Then there is a PL homeomorphism $f: A \rightarrow A$ such that $f \mid \partial A=\mathrm{id}$ and $C \subset f U$.

Proof. We may assume that $A=I^{k}$ and that $B$ is an ordinary face of $I^{k}$, in which case the lemma is elementary.

2.3. Encasing lemma. Let $M^{n}$ be a C-manifold and $X^{k} a$ TOP submanifold. Let $D_{1}, D_{2} \subset X$ be closed subsets which are C-encasable in $(M, X)$. Then $D_{1} \cup D_{2}$ is also $C$-encasable in $(M, X)$ if either

(i) $k \neq 4, D_{2}$ is a $k$-cell which is locally flat in int $X$ and $D_{1} \cap D_{2}$ is a (k-1)cell locally flat in $\partial D_{2}$ or

(ii) $k \neq 5, D_{2}$ is a $(k-1)$-cell which is locally flat in $\partial X$ and $D_{1} \cap D_{2}$ is a $(k-2)$ cell locally flat in $\partial D_{2}$.

In parallel, let $g: Y \rightarrow M$ be an embedding, where $Y \subset \bar{R}^{k}$. Let $D_{1}, D_{2} \subset X=g Y$ be closed subsets which are $(C, g)$-encasable. Then $D_{1} \cup D_{2}$ is $(C, g)$-encasable if either

(iii) $g^{-1} D_{2}$ is a PL $k$-cell in int $Y$ and $g^{-1}\left[D_{1} \cap D_{2}\right]$ is a PL $(k-1)$-cell in $\partial g^{-1} D_{2}$ or

(iv) $g^{-1} D_{2}$ is a PL $(k-1)$-cell in $\partial Y$ and $g^{-1}\left[D_{1} \cap D_{2}\right]$ is a PL $(k-2)$-cell in $\partial g^{-1} D_{2}$.

Proof. Only (i) and (iii) are considered, the proofs of (ii) and (iv) being somewhat similar.

Let $h_{i}: U_{i} \rightarrow M$ be $C$-encasings or $(C, g)$-encasings of $D_{i}(i=1,2)$ with $h_{2}^{-1} X=$ = $U_{2} \cap \bar{R}^{k}$. Of course in case (i), $U_{i}=B^{n}$.

Choose a PL $k$-cell $A$ and a PL $(k-1)$-cell $B$ so that: $h_{\mathrm{a}}^{-1} D_{2} \subset$ int $A ; A \subset U_{2} \cap \bar{R}^{k}$; $B \subset \partial A \cap h_{2}^{-1} h_{1} U_{1} ; \partial A \cap h_{2}^{-1} D_{1} \subset$ int $B$. In case (i), local flatness of $D_{1} \cap D_{2}$ in $\partial D_{2}$ and of $D_{2}$ in int $X$ provide a topological cell pair as $A$ and $B$. Obtain the PL pair by use of PL approximation, see 1.2. In case (iii), the existence of $A$ and $B$ follows immediately from the hypotheses, since $h_{2}^{-1} h_{1}=$ id in $\bar{R}^{k} \cap U_{1} \cap U_{2}$.

Let $f: A \rightarrow A$ be the PL homeomorphism given by 2.2 with $C=A \cap h_{2}^{-1}\left[D_{1} \cup D_{2}\right]$ and $U=A \cap h_{2}^{-1} h_{1} U_{1}$. Extend $f$ by suspension to a PL homeomorphism of a PL $n$-cell $A^{*} \subset U_{2}$, and further extend this to a PL homeomorphism $\varphi$ of $R^{n}$, where $\varphi=\mathrm{id}$ on $R^{n} \backslash A^{*}$. 
Now define the map $h: \varphi U_{1} \rightarrow M$ by setting $h=h_{1} \varphi^{-1}$ in $\varphi\left[U_{1} \backslash h_{1}^{-1} h_{2} A^{*}\right]$ and $h=h_{2} \varphi h_{2}^{-1} h_{1} \varphi^{-1}$ in $\varphi h_{1}^{-1} h_{2} A^{*}$. In case (i), $h$ is a $C$-encasing of $D_{1} \cup D_{2}$ in $(M, X)$ and in case (iii), $h$ is a $(C, g)$-encasing of $D_{1} \cup D_{2}$. Note that in case (i), $h$ could have been $h_{1}$ in $U \backslash h_{1}^{-1} h_{2} A^{*}$ and $h_{2} \varphi h_{2}^{-1} h_{1}$ in $h_{1}^{-1} h_{2} A^{*}$.

2.4. Relative Schoenflies theorem. Let $1 \leqq l \leqq k \leqq n$, let $Y$ be either $\emptyset, R^{k}$ or $R_{+}^{k}$, let $Z$ be either $\emptyset$ or $Y \cap R^{l}$, and let $A=\bar{B}^{n} \backslash B^{n}(1 / 2)$. Suppose $e: A \rightarrow R^{n}$ is a $C$-embedding such that $e \mid A \cap Z$ is the inclusion, $e^{-1}(Y, Z)=A \cap(Y, Z)$, and $e A \subset \bar{G}$, where $G$ is the bounded component of $R^{n} \backslash e S^{n-1}$. Then there is a $C$-homeomorphism $\hat{e}:\left(\bar{B}^{n}, \bar{B}^{n} \cap Y\right) \rightarrow(\bar{G}, \bar{G} \cap Y)$ such that $\hat{e}=e$ on $S^{n-1}$ and $\hat{e} \mid \bar{B}^{n} \cap Z$ is the inclusion.

Proof. If $n=1$, the theorem is almost trivial for $C=$ TOP or LIP but not for $C=\mathrm{LQC}$. In this case we may apply the method of [LeV 1, p. 12] to replace $e$ by a quasisymmetric function continuously differentiable on $B^{1}(3 / 4) \backslash B^{1}(1 / 2)$. The sought-for $\hat{e}$ can then be chosen to be PL in a neighbourhood of $[-1 / 2,1 / 2]$.

Suppose that $n \geqq 2$. By going through the non-relative version $Y=\emptyset$ in $[\mathrm{GV}$, Theorem 3], one can ensure that all of the auxiliary maps can be chosen to setwise respect $Y$ and $Z$. Although the proof in [GV] is stated for the categories QC and TOP, it is equally valid in LIP. Thus 2.4 is true for $Z=\emptyset$. Assume $Z \neq \emptyset$. If $C$ is LIP or TOP, one can replace a $C$-homeomorphism $\hat{e}:\left(\bar{B}^{n}, \bar{B}^{n} \cap Y, \bar{B}^{n} \cap Z\right) \rightarrow$ $(\bar{G}, \bar{G} \cap Y, \bar{G} \cap Z)$ with $\hat{e}=e$ on $S^{n-1}$ by $\hat{e} \sigma^{-1}$, where $\sigma$ is the $(n-l)$ th suspension of $\hat{e} \mid \bar{B}^{n} \cap Z$ (firstly, in the case $Z=R_{+}^{k}$, extended over $\bar{B}^{k}$ by reflection in $\bar{B}^{k-1}$ ), to obtain the desired $C$-homeomorphism.

For $C=\mathrm{LQC}$, the above suspension need not be a $C$-homeomorphism, so one must return to the proof in [GV] with $Z$ in mind. We will use the notation of $[\mathrm{GV}, \S 5]$.

We begin with Lemma 7. With $e$ as above and $G$ playing the role of $C$ in Lemma 7, we claim that the $C$-embedding $f: \bar{B}^{n} \rightarrow \bar{R}^{n}$ may be chosen so that $f \bar{B}^{n} \cup e[$ int $A]=G$, that $f^{-1} Y=\bar{B}^{n} \cap Y$ and that $f \mid \bar{B}^{n}(3 / 4) \cap Z=\mathrm{id}$. To ensure this, one carries out the construction in Lemma 7 replacing $1 / 2$ by $3 / 4$ thus in effect considering only $e \mid \bar{B}^{n} \backslash B^{n}(3 / 4)$. Since the initial conformal normalisation may destroy the fact that $e \mid A \cap Z=\mathrm{id}$, we must forgo that luxury. Instead, choose a QC homeomorphism $f^{\prime}: \bar{R}^{n} \rightarrow \bar{R}^{n}$ which satisfies the conditions: $f^{\prime} \mid \bar{B}^{n}(3 / 4)=\mathrm{id}, f^{\prime} Y=Y, f^{\prime}(\infty)=x_{0}=$ $7 e_{1} / 8$, and $\bar{R}^{n} \backslash f^{\prime} B^{n}$ is a small ball centred at $x_{0}$ and contained in $e\left[B^{n} \backslash \bar{B}^{n}(3 / 4)\right]$. In the construction of Lemma 7 we replace $1 / 2$ by $3 / 4$ and choose the auxiliary map $\beta$ so that $\beta\left(x_{0}\right)=0$. We thus obtain an LQC embedding $\bar{f}: \bar{R}^{n} \backslash\left\{x_{0}\right\} \rightarrow \bar{R}^{n}$ defined either as $e \alpha^{-1} \beta \alpha e^{-1}$ or the identity. Corresponding to the map $f$ of Lemma 7 we obtain an LQC embedding $f_{1}: \bar{R}^{n} \backslash\left\{x_{0}\right\} \rightarrow \bar{R}^{n}$ defined as $\bar{f}^{-1} \gamma \bar{f}$ or the identity. Then $f=f_{1} f^{\prime}$ is the desired map.

Let $\delta: \bar{B}^{n}(3 / 4) \rightarrow \bar{B}^{n}$ be a QC embedding which is the identity on $\bar{B}^{n}(3 / 4) \cap Z$, respects $Y$ and is such that $\delta B^{n}(3 / 4) \cup f^{-1} e[$ int $A]=\bar{B}^{n}$ and $\delta B^{n}(1 / 3) \cap f^{-1} e A=\emptyset$. The map $\delta$ could be chosen so that $\bar{B}^{n} \backslash \delta \bar{B}^{n}(3 / 4)$ is a small neighbourhood of 
$\left(\bar{B}^{n} \backslash \bar{B}^{n}(3 / 4)\right) \cap Z$ in $\bar{B}^{n}$, and $\delta B^{n}(1 / 3)$ is a small ellipsoidal neighbourhood of $B^{n}(1 / 3) \cap Z$. The QC embedding $g=f \delta: \bar{B}^{n}(3 / 4) \rightarrow R^{n}$ satisfies:

(a) $g B^{n}(3 / 4) \cup e[$ int $A]=G$;

(b) $g\left[\bar{B}^{n}(3 / 4) \backslash B^{n}(b)\right] \subset e\left[B^{n} \backslash \bar{B}^{n}(a)\right]$ for some $a, b$ with $1 / 2<a \leqq b<3 / 4$;

(c) $g B^{n}(1 / 3) \cap e A=\emptyset$;

(d) $g \mid \bar{B}^{n}(3 / 4) \cap Z=$ id;

(e) $g^{-1} Y=\bar{B}^{n}(3 / 4) \cap Y$.

Inclusion (b) certainly holds with $a=1 / 2, b=3 / 4$, so it also holds for some $a>1 / 2$, $b<3 / 4$. The extra condition $a \leqq b$ ensures that $a / 3 b \leqq 1 / 3$, which is needed when considering $\tilde{e} \lambda^{-1} \mu^{-1} \mid S^{n-1}(a / 3 b)$.

We now apply the procedure of Lemma 9 of $[\mathrm{GV}]$ to the QC embedding $h=g^{-1} e: e^{-1} g \bar{B}^{n}(3 / 4) \rightarrow R^{n}$. Define radial QC homeomorphisms $\lambda, \mu: R^{n} \rightarrow R^{n}$ so that: $\lambda$ is the identity on $R^{n} \backslash B^{n}(3 / 4)$, expands $\bar{B}^{n}(3 / 4) \backslash B^{n}(b)$ onto $\bar{B}^{n}(3 / 4) \backslash B^{n}(1 / 3)$ and is division by $3 b$ on $\bar{B}^{n}(b) ; \mu$ is multiplication by $a$ on $R^{n} \backslash B^{n}$, contracts $\bar{B}^{n} \backslash B^{n}(1 / 2)$ onto $\bar{B}^{n}(a) \backslash B^{n}(1 / 2)$ and is the identity on $\bar{B}^{n}(1 / 2)$.

Define the QC embedding $\tilde{e}: \bar{B}^{n} \backslash B^{n}(a) \rightarrow R^{n}$ by

$$
\tilde{e}(x)= \begin{cases}h \mu(x) & \text { if } x \notin e^{-1} g \bar{B}^{n}(3 / 4), \\ \lambda h(x) & \text { if } g \lambda h(x) \notin e A, \\ h \mu h^{-1} \lambda h(x) & \text { otherwise. }\end{cases}
$$

As in the case of $\bar{e}$ in the proof of Lemma 9 of [GV], $\tilde{e}$ is well-defined and, using properties of $g$, one can verify that $\tilde{e}$ acts in the same way on $S^{n-1}$ as on $S^{n-1}(a)$, more precisely, $\tilde{e}(x)=h(a x)$ if $x \in S^{n-1}$ and $\tilde{e}(x)=h(x) / 3 b$ if $x \in S^{n-1}(a)$. Property (e) of $g$ ensures that $\tilde{e}^{-1} Y=\left(\bar{B}^{n} \backslash B^{n}(a)\right) \cap Y$, but $\tilde{e}$ is not necessarily the inclusion in $Z$. Indeed, $\tilde{e}=\mu \lambda$ on $\left(\bar{B}^{n} \backslash B^{n}(a)\right) \cap Z$. Hence the QC embedding $\tilde{e} \lambda^{-1} \mu^{-1}: \bar{B}^{n}(a) \backslash B^{n}(a / 3 b) \rightarrow R^{n}$ is the inclusion in $Z$, still respects $Y$, and acts in the same way on $S^{n-1}(a)$ as on $S^{n-1}(a / 3 b)$. As in the proof of Lemma 9 of [GV], exploit this feature of $\tilde{e} \lambda^{-1} \mu^{-1}$ to extend it to a QC embedding $e^{\prime}: \bar{B}^{n}(a) \rightarrow R^{n}$. Since $g e^{\prime}=e$ on $S^{n-1}(a), g e^{\prime}$ extends to a QC embedding $\hat{e}: \bar{B}^{n} \rightarrow R^{n}$ having all of the required properties.

\section{Flattening spheres}

In this section we consider a TOP $k$-sphere $X \subset S^{n}$ which is locally $C$-flat in $S^{n}$. If $k=n-2 \geqq 1$, then $X$ may be knotted, so we must add the hypothesis that $S^{n} \backslash X$ is homotopy equivalent to $S^{1}$. We shall prove in 3.3 that, unless $k=4$ or $(n, k)=(4,2), X$ is $C$-flat in $S^{n}$ in the sense that $\left(S^{n}, X\right)$ is $C$-homeomorphic to $\left(S^{n}, S^{k}\right)$. 
A similar result holds for locally $C$-flat embeddings, and in 3.4 we prove that, unless $(n, k)=(4,2)$, any locally $C$-flat embedding $g: \bar{R}^{k} \rightarrow \bar{R}^{n}$ extends to a $C$-homeomorphism of $\bar{R}^{n}$. Of course, as above, we require $g \bar{R}^{k}$ to be homotopically unknotted.

Finally we show that our methods adapt to verifying uniqueness of $C$-structures on $R^{n}$ and $S^{n}$ when $n \neq 4$ and on $I^{n}$ when $n \neq 4$ or 5 .

3.1. Lemma. Let $X \subset S^{n}$ be a TOP $k$-sphere and let $h_{1}, h_{2}: B^{n} \rightarrow S^{n}$ be $C$-encasings in $\left(S^{n}, X\right)$ such that $S^{n}=h_{1} B^{n} \cup h_{2} B^{n}$. Then $\left(S^{n}, X\right)$ is C-homeomorphic to $\left(S^{n}, S^{k}\right)$.

Proof. We replace $S^{n}$ by $\bar{R}^{n}$. Choose $r \in(0,1)$ so that $B^{n} \backslash B^{n}(r) \subset h_{1}^{-1} h_{2} B^{n}$. Applying the relative Schoenflies theorem 2.4, with $Z=\emptyset$, together with an inversion we find a $C$-homeomorphism $f$ of $\bar{R}^{n} \backslash B^{n}(r)$ onto the closure of the bounded component of $R^{n} \backslash h_{2}^{-1} h_{1} S^{n-1}(r)$ such that $f^{-1} B^{k}=\bar{R}^{k} \backslash B^{k}(r)$ and $f=h_{2}^{-1} h_{1}$ on $S^{n-1}(r)$. The sought-for $C$-homeomorphism $h:\left(\bar{R}^{n}, \bar{R}^{k}\right) \rightarrow\left(\bar{R}^{n}, X\right)$ is obtained by setting $h=h_{1}$ in $B^{n}(r)$ and $h=h_{2} f$ in $\bar{R}^{n} \backslash B^{n}(r)$.

3.2. Lemma. Let $X \subset \Sigma^{n}$ be a TOP $k$-sphere and let $h_{1}, h_{2}: B^{n} \rightarrow \Sigma^{n}$ be $C$-encasings in $\left(\Sigma^{n}, X\right)$ such that $X=h_{1} B^{k} \cup h_{2} B^{k}$. Suppose that $n \neq 4$ and there are a homeomorphism $\alpha: \Sigma^{n} \rightarrow \Sigma^{n}$ and a PL $k$-cell $D_{1} \subset \Sigma^{k}$ so that $\alpha \Sigma^{k}=X$, $D_{1} \subset \alpha^{-1} h_{1} B^{k}$ and $\Sigma^{k} \backslash$ int $D_{1}=D_{2} \subset \alpha^{-1} h_{2} B^{k}$. Then there is a C-homeomorphism $\varphi: \Sigma^{n} \rightarrow \Sigma^{n}$ such that $\varphi \mid X=\mathrm{id}$ and $\varphi h_{1} B^{n} \cup h_{2} B^{n}=\Sigma^{n}$.

Proof. Choose $r \in(0,1)$ so that $D_{i} \times I^{n-k}(r) \subset \alpha^{-1} h_{i} B^{n}$, and a PL homeomorphism $\beta: I^{k+1} \times \Sigma^{n-k-1}(r) \rightarrow T=\operatorname{cl}\left(\Sigma^{n} \backslash \Sigma^{k} \times I^{n-k}(r)\right)$ such that $\beta=\mathrm{id}$ on $\Sigma^{k} \times \Sigma^{n-k-1}(r)$. Next choose neighbourhoods $U_{i}$ of $D_{i}$ in $I^{k+1}$ such that $\bar{U}_{i} \times \Sigma^{n-k-1}(r) \subset \beta^{-1} \alpha^{-1} h_{i} B^{n}$.

By 1.2, we can choose a PL homeomorphism $\gamma$ approximating $\alpha \mid T$ such that $\gamma \beta\left[\bar{U}_{i} \times \Sigma^{n-k-1}(r)\right] \subset h_{i} B^{n}$ and $X \cap \gamma T=\emptyset$. By Lemma 2.2, there is a PL homeomorphism $\delta: I^{k+1} \rightarrow I^{k+1}$ such that $\delta \mid \Sigma^{k}=$ id and $\delta U_{1} \cup U_{2}=I^{k+1}$. Then $\gamma \beta(\delta \times$ id $) \beta^{-1} \gamma^{-1}$ is a PL self homeomorphism of $\gamma T$ which can be extended by the identity to a PL homeomorphism $\varphi: \Sigma^{n} \rightarrow \Sigma^{n}$ with the desired properties.

3.3. Theorem. Suppose that $X \subset S^{n}$ is a locally $C$-flat TOP $k$-sphere and that $k \neq 4$ and $(n, k) \neq(4,2)$. If $k=n-2$, assume also that $S^{n} \backslash X$ is homotopy equivalent to $S^{1}$. Then $\left(S^{n}, X\right)$ is C-homeomorphic to $\left(S^{n}, S^{k}\right)$.

Proof. Choose a homeomorphism $\alpha: \bar{R}^{k} \rightarrow X$ and let $h_{2}: B^{n} \rightarrow S^{n}$ be a $C$-encasing of $\alpha(\infty)$ in $\left(S^{n}, X\right)$. Let $Q=I^{k}(r)$ be a $k$-cube containing $R^{k} \backslash \alpha^{-1} h_{2} B^{k}$ in its interior. Subdivide $Q$ in the natural way into cubes $Q_{1}, \ldots, Q_{m}$ such that each $\alpha Q_{j}$ is $C$-encasable in $\left(S^{n}, X\right)$ and $Q_{j+1} \cap\left(Q_{1} \cup \ldots \cup Q_{j}\right)$ is a $\operatorname{PL}(k-1)$-cell in $\partial Q_{j+1}$ for $1 \leqq j \leqq m-1$. Applying Lemma 2.3 (i) inductively we see that $\alpha Q$ is $C$-encasable in $\left(S^{n}, X\right)$; let $h_{1}: B^{n} \rightarrow S^{n}$ be a $C$-encasing of $\alpha Q$ in $\left(S^{n}, X\right)$.

We consider three cases separately: $n-k \geqq 3 ; n \neq 4 ;(n, k)=(4,3)$. 
Firstly suppose $n-k \geqq 3$. Replace $S^{n}$ by $\bar{R}^{n}$. Assume for the moment that $h_{2}=\varrho \mid B^{n}$, where $\varrho: \bar{R}^{n} \rightarrow \bar{R}^{n}$ is reflection in $S^{n-1}$. Since $X \cap \bar{B}^{n} \subset h_{1} B^{n}$, we may assume that $X \cap \bar{B}^{n}(2) \subset h_{1} B^{n}$. In the notation of [Ru, Lemma 4.5.2, p. 159], set $M=R^{n} \backslash\left(R^{k} \backslash B^{k}(2)\right), U=h_{1} B^{n} \backslash\left(R^{k} \backslash B^{k}(2)\right), C=B^{k}(2) \backslash B^{k}$ and $P=B^{k}(2)$. From this lemma it follows that there is a $C$-homeomorphism of $M$ having compact support, so that it extends to a $C$-homeomorphism $\beta: \bar{R}^{n} \rightarrow \bar{R}^{n}$, with $\bar{B}^{k}(2) \subset \beta h_{1} B^{n}$. Choose $s<1$ large enough so that $\bar{B}^{k}(2) \subset \beta h_{1} B^{n}(s)$. Then $\left(\beta h_{1} \mid B^{n} \backslash B^{n}(s)\right)^{-1} \bar{R}^{k}=B^{k} \backslash B^{k}(s)$, so by the relative Schoenflies theorem 2.4 with $Z=\emptyset, \beta h_{1} \mid \bar{B}^{n}(s)$ extends to a $C$-homeomorphism $\gamma: \bar{R}^{n} \rightarrow \bar{R}^{n}$ with $\gamma \bar{R}^{k}=\beta X$. Hence $\beta^{-1} \gamma$ is a $C$-homeomorphism of $\bar{R}^{n}$ taking $\bar{R}^{k}$ onto $X$. If $h_{2} \neq \varrho \mid B^{n}$, then, using the $C$-Schoenflies theorem, we can assume that $h_{2}$ extends to a $C$-homeomorphism, call it $h_{2}$ also, of $\bar{R}^{n}$. Observe that $\varrho h_{2}^{-1} h_{1}: B^{n} \rightarrow \bar{R}^{n}$ and $\varrho: B^{n} \rightarrow \bar{R}^{n}$ are $C$-encasings in $\left(\bar{R}^{n}, \varrho h_{2}^{-1} X\right)$ with images covering $\varrho h_{2}^{-1} X$, so that by what we have just shown, $\left(\bar{R}^{n}, \varrho h_{2}^{-1} X\right)$ is $C$-homeomorphic to $\left(\bar{R}^{n}, \bar{R}^{k}\right)$. Since $\varrho h_{2}^{-1}$ is a $C$-homeomorphism from $\left(\bar{R}^{n}, X\right)$ to $\left(\bar{R}^{n}, \varrho h_{2}^{-1} X\right)$, this completes the case where $n-k \geqq 3$.

Secondly consider the case $n \neq 4$. Replacing $\bar{R}^{n}$ and $S^{n}$ by $\Sigma^{n}$, we see that $\alpha: \Sigma^{k} \rightarrow X$ extends to a homeomorphism $\alpha:\left(\Sigma^{n}, \Sigma^{k}\right) \rightarrow\left(\Sigma^{n}, X\right)$. For $(n, k) \neq(3,1)$, this extension follows from [Ru, p. 48 and p. 158]; for $(n, k)=(3,1)$ from Dehn's lemma [Mo 2, p. 199] and from [Bi]. With $D_{1}=Q$, the hypotheses of Lemma 3.2 are satisfied: let $\varphi: \Sigma^{n} \rightarrow \Sigma^{n}$ be the $C$-homeomorphism given by 3.2. Then $\varphi h_{1}$ is a $C$-encasing in $\left(\Sigma^{n}, X\right)$ with $\varphi h_{1} B^{n} \cup h_{2} B^{n}=\Sigma^{n}$. The assertion now follows from 3.1.

Finally suppose $(n, k)=(4,3)$. Choose $s \in(0,1)$ so that $h_{1}^{-1} \alpha Q \subset B^{3}(s)$. By the Schoenflies theorem 2.4 (with $Y=\emptyset$ ) we can extend $h_{2}^{-1} h_{1} \mid S^{3}(s)$ to a $C$-embed$\operatorname{ding} f: \bar{R}^{3} \backslash B^{3}(s) \rightarrow B^{3}$. Define $u: \bar{R}^{3} \rightarrow S^{4}$ by $u=h_{1}$ on $B^{3}(s)$ and $u=h_{2} f$ on $\bar{R}^{3} \backslash B^{3}(s)$. Then $u$ is an embedding and $u \bar{R}^{3}=X$. If $C=$ LIP, $u$ is a LIP embedding, and the theorem follows from [LuV, 7.8]. Suppose $C=\mathrm{LQC}$. By [GV, Corollary 5, p. 230], it suffices to show that $u$ is locally QC flat. This is clearly true at points of $B^{3}(s)$, since $h_{1} \mid B^{4}(s)$ is a $\mathrm{QC}$ extension of $u \mid B^{3}(s)$. If $x \in \bar{R}^{3} \backslash B^{3}(s)$, we apply Carleson's extension theorem [Ca], see also 3.12, to find a QC embedding $v$ of a neighbourhood $U$ of $x$ in $\bar{R}^{4}$ such that $v=h_{2}^{-1} u$ in $U \cap \bar{R}^{3}$. Then $h_{2} v$ is a QC local extension of $u$ at $x$.

3.4. Theorem. Let $g: \bar{R}^{k} \rightarrow \bar{R}^{n}$ be a locally $C$-flat embedding with $(n, k) \neq(4,2)$. If $k=n-2$, assume that $\bar{R}^{n} \backslash g \bar{R}^{k}$ is homotopy equivalent to $S^{1}$. Then $g$ extends to a C-homeomorphism of $\bar{R}^{n}$.

Proof. Proceeding as in the proof of 3.3 with $\alpha=g$, we may use Lemma 2.3 (iii) to reduce the number of $(C, g)$-encasings to two.

The proof in the case $(n, k)=(4,3)$ may now proceed just as in the corresponding part of 3.3. In the case $n \neq 4$, we also proceed much as in the corresponding part of 3.3 , observing that the homeomorphism $\varphi$ of 3.2 is the identity on $g \bar{R}^{k}$, so that we have two $(C, g)$-encasings $h_{1}: B^{n}(b) \rightarrow \bar{R}^{n}$ and $h_{2}: \bar{R}^{n} \backslash \bar{B}^{n}(a) \rightarrow \bar{R}^{n}$ for some $a<b$ so that $\operatorname{im} h_{1} \cup \operatorname{im} h_{2}=\bar{R}^{n}$. The result now follows from the $(C, g)$-version of 
Lemma 3.1. This version is proved just as the original except that the appeal to the relative Schoenflies theorem uses the case $Z=Y$.

In the case $n-k \geqq 3$, we again follow the proof of the appropriate part of 3.3. We begin with two $(C, g)$-encasings $h_{1}: B^{n}(b) \rightarrow \bar{R}^{n}(b>2)$ and id: $\bar{R}^{n} \backslash \bar{B}^{n} \rightarrow \bar{R}^{n}$, engulfing via $\beta: \bar{R}^{n} \rightarrow \bar{R}^{n}$ as before and using the $Z=Y$ version of the relative Schoenflies theorem to extend $\beta h_{1} \mid B^{n}(2)$ to the $C$-homeomorphism $\gamma: \bar{R}^{n} \rightarrow \bar{R}^{n}$ with $\gamma \mid \bar{R}^{k} \backslash B^{k}(2)=$ id. The $C$-homeomorphism $\beta^{-1} \gamma: \bar{R}^{n} \rightarrow \bar{R}^{n}$ extends $g$. If the second $\left(C, g\right.$ )-encasing is $h_{2}: \bar{R}^{n} \backslash \bar{B}^{n} \rightarrow \bar{R}^{n}$, not necessarily the identity, then as in 3.3 we extend it to a $C$-homeomorphism $h_{2}: \bar{R}^{n} \rightarrow \bar{R}^{n}$, extend $h_{2}^{-1} g: \bar{R}^{k} \rightarrow \bar{R}^{n}$ to a $C$-homeomorphism of $\bar{R}^{n}$ using the previous part and then follow this by $h_{2}$ to get the desired extension of $g$.

3.5. Remark. The missing case $(n, k)=(4,2)$ in Theorems 3.3 and 3.4 seems to be difficult: one needs a version of Lemma 3.2 for this case, the other steps in the proofs of 3.3 and 3.4 being valid in this case. It is of interest to note that the two questions concerning 3.3 and 3.4 arising from this case are equivalent. To see this, one proceeds much as in the last paragraph of the proof of 3.3, but appealing to Ahlfors' extension theorem [Ah] to extend a QC map from $\bar{R}^{2}$ to $\bar{R}^{4}$.

3.6. Theorem. Let $X$ be a locally LIP flat LIP $k$-sphere in $S^{n},(n, k) \neq(4,2)$. If $k=n-2$, assume also that $S^{n} \backslash X$ is homotopy equivalent to $S^{1}$. Then $\left(S^{n}, X\right)$ is lipeomorphic to $\left(S^{n}, S^{k}\right)$.

Proof: Let $g: \bar{R}^{k} \rightarrow X$ be a lipeomorphism. By 3.4, it suffices to show that $g$ is locally LIP flat at each point $a \in \bar{R}^{k}$. We may assume $a \neq \infty$. Choose a LIP encasing $h: B^{n} \rightarrow S^{n}$ of $g(a)$ in $\left(S^{n}, X\right)$. Then $h\left(h^{-1} g \times\right.$ id $)$ is a local extension of $g$, defined in a neighbourhood of $a$ in $R^{n}$.

3.7. One-sided versions. Let $X$ be a TOP $(n-1)$-sphere in $S^{n}$ and let $G$ be a component of $S^{n} \backslash X$. Modifying in an obvious way the definitions in 2.1 we define local $C$-flatness of $X$ in $\bar{G}$ and local $C$-flatness of an embedding $g: \bar{R}^{n-1} \rightarrow S^{n}$, $g \bar{R}^{n-1}=X$, in $\bar{G}$. An easy modification of the preceding proofs then yields the following results, the second of which was essentially proved in [GV] for $C=\mathrm{LQC}$ and in $[\mathrm{LuV}]$ for $C=\mathrm{LIP}$.

3.8. Theorem. Let $n \neq 5$, let $X \subset S^{n}$ be a TOP $(n-1)$-sphere, and let $G$ be a component of $S^{n} \backslash X$. If $X$ is locally $C$-flat in $\bar{G}$, then $\bar{G}$ is $C$-homeomorphic to $\bar{B}^{n}$.

3.9. Theorem. Let $g: \bar{R}^{n-1} \rightarrow \bar{R}^{n}$ be an embedding which is locally $C$-flat in $\bar{G}$, where $G$ is a component of $\bar{R}^{n} \backslash g \bar{R}^{n-1}$. Then $g$ can be extended to a C-homeomorphism $g^{*}: \bar{R}_{+}^{n} \rightarrow \bar{G}$.

3.10. Uniqueness of C-structures. In [LuV, 8.3-8.5] it was proved that the manifolds $R^{n}$ and $S^{n}$ have an essentially unique LIP structure for $n \neq 4$, and that 
the same is true for $I^{n}, n \neq 4,5$. (Much more general results have been obtained by Sullivan [Su].) The essential step in the proof of these results $[\mathrm{LuV}, 8.3]$ consisted of showing that if $M$ is a LIP manifold homeomorphic to $R^{n}, n \neq 4$, then every compact set $A \subset M$ is contained in a LIP ball. The proof was based on a LIP version of Newman's engulfing theorem. We remark that this can also be easily proved for both $C=\mathrm{LIP}$ and $C=\mathrm{LQC}$ using the encasing lemma 2.3. Indeed we can again cover $A$ with a TOP $n$-cube, divide this into small cubes each of which is $C$-encasable in $(M, M)$ and then use 2.3 to show that $A$ is $C$-encasable in $(M, M)$. Thus we obtain the following result:

3.11. Theorem. Let $M$ be $R^{n}$ or $S^{n}, n \neq 4$ or $I^{n}, n \neq 4,5$. Let $M_{1}$ be a C-manifold homeomorphic to $M$. Then $M_{1}$ is C-homeomorphic to $M$.

3.12. Remark on Carleson's proof. In the proof of 3.3 we made use of Carleson's extension theorem [Ca]: Every QC homeomorphism $f: R^{3} \rightarrow R^{3}$ can be extended to a QC homeomorphism $F: R^{4} \rightarrow R^{4}$. This will also be needed in 4.1. The ingenious proof in [Ca] is rather sketchy and difficult to follow because of a number of misprints. The most serious misprint is on p. 43 , where the extension map $F$ is defined in $R^{3} \times\left[2^{-m(v+1)}, 2^{-m v}\right]$ by the formula

$$
F(x ; y)=\left(f_{v}\left(x ; 2^{m(v+1)} y^{\prime}\right) ; \varrho_{v+1}\left(f_{v+1}(x)\right)+y^{\prime} 2^{m(v+1)}\left(\varrho_{v}\left(f_{v}(x)\right)-\varrho_{v+1}\left(f_{v+1}(x)\right)\right)\right),
$$

where $y^{\prime}=y-2^{-m(v+1)}$. First, it is clear that the isotopy $f_{v}(x, t)$ should be from $f_{v+1}$ to $f_{v}$ and not vice versa. Secondly, the constant $2^{m(v+1)}$ should be replaced by $\left(2^{-m v}-2^{-m(v+1)}\right)^{-1}=2^{m(v+1)} /\left(2^{m}-1\right)$. However, even with these corrections, the method of estimating $D F$ from below is incorrect. In fact, we have not even been able to show that $F$ is injective.

We suggest that (3.13) be replaced by the formula

$$
F(x ; y)=\left(f_{v}(x ; t) ; t \varrho_{v}\left(f_{v}(x ; t)\right)+(1-t) \varrho_{v+1}\left(f_{v}(x ; t)\right)\right),
$$

where $t=q\left(y-2^{-m(v+1)}\right), q=2^{m(v+1)} /\left(2^{m}-1\right)$, and $f_{v}(x ; t)$ is the isotopy from $f_{v+1}$ to $f_{v}$. Indeed, we can now find an explicit expression for $G=F^{-1}$ as follows: Let $u \mapsto A(u ; t)$ be the inverse map of $x \mapsto f_{v}(x ; t)$. Then $G(u ; v)=\left(A(u ; t) ; 2^{-m(v+1)}+t / q\right)$ where $t=t(u ; v)=\left(v-\varrho_{v+1}(u)\right) /\left(\varrho_{v}(u)-\varrho_{v+1}(u)\right)$. Then direct computations yield the estimate

$$
|D G(u ; v)| \leqq 2^{-m v} C / \varrho_{v}(u) .
$$

On the other hand, as in [Ca] we obtain

$$
|D F(x ; y)| \leqq 2^{--m v} C \varrho_{v}\left(f_{v}(x ; t)\right) .
$$

These inequalities imply that $F$ is quasiconformal. 


\section{Flattening cells}

In this section we apply the methods of the previous sections to $C$-flatten cells and embeddings onto cells in $S^{n}$. When we combine $C$-encasings, we must be sure that the boundary is preserved. For this reason we require an additional dimensional restriction on the cell.

4.1. Lemma. Let $E_{1}=I^{k}, E_{2}=I^{k}+2 e_{k}, E=E_{1} \cup E_{2}$, and let $X \subset \bar{R}^{n}$ be the union of two $k$-cells $D_{1}, D_{2}$ such that $\left(X ; D_{1}, D_{2}\right)$ is homeomorphic to $\left(E ; E_{1}, E_{2}\right)$.

(i) If $D_{1}$ and $D_{2}$ are $C$-encasable in $\left(\bar{R}^{n}, X\right)$, then $\left(\bar{R}^{n}, X\right)$ is $C$-homeomorphic to $\left(\bar{R}^{n}, \bar{B}^{k}\right)$.

(ii) If $g: E \rightarrow \bar{R}^{n}$ is an embedding so that $g\left(E ; E_{1}, E_{2}\right)=\left(X ; D_{1}, D_{2}\right)$ and $D_{1}$ and $D_{2}$ are $(C, g)$-encasable, then $g$ extends to a self $C$-homeomorphism of $\bar{R}^{n}$.

Proof. (i) We present two proofs, the first valid for $n \neq 4$ and all $k$ and the second valid for $k \neq 5$ and all $n$ if $C=\mathrm{LIP}$ and for $n \leqq 4$ if $C=\mathrm{LQC}$.

Assume, then, that $n \neq 4$, and let $h_{i}: B^{n} \rightarrow \bar{R}^{n}$ be $C$-encasings of $D_{i}$ in $\left(\bar{R}^{n}, X\right)$. Since $X$ is locally flat in $\bar{R}^{n}$, by [Ru, p. 106] there is a homeomorphism $f:\left(\bar{R}^{n}, E\right) \rightarrow\left(\bar{R}^{n}, X\right)$. Using an auxiliary homeomorphism of $\bar{R}^{k}$, we may choose $f$ so that $f E_{i}=D_{i}$. Replacing $\bar{R}^{n}$ by $\Sigma^{n}$ for the moment, since $n \neq 4$, we may by 1.2 approximate $f$ by PL homeomorphisms and hence find a PL $n$-cell $A$ and a PL $(n-1)$-cell $B$ such that $X \subset \Sigma^{n} \backslash A, \operatorname{cl}\left(\Sigma^{n} \backslash A\right) \subset h_{1} B^{n} \cup h_{2} B^{n}, B \subset \partial A \cap h_{2} B^{n}$ and $\operatorname{cl}(\partial A \backslash B) \subset h_{1} B^{n}$. Applying Lemma 2.2 with $U=A \cap h_{2} B^{n}$ and $C=A \cap h_{1} B^{n}$ we find a PL homeomorphism $h: \Sigma^{n} \rightarrow \Sigma^{n}$ such that $h \mid \Sigma^{n} \backslash A=$ id and $h h_{2} B^{n} \cap h_{1} B^{n}=\Sigma^{n}$. Replacing $\Sigma^{n}$ by $\bar{R}^{n}$ and $h_{2}$ by $h h_{2}$ allows us to assume that $h_{1} B^{n} \cap h_{2} B^{n}=\bar{R}^{n}$. The proof in this case may now be completed as in 3.1 using the relative Schoenflies theorem 2.4 with $Y=R_{+}^{k}$.

Next assume that $k \neq 5$ and, if $C=\mathrm{LQC}, n \leqq 4$. Let $h_{i}: B^{n} \rightarrow \bar{R}^{n}$ be $C$-encasings of $D_{i}$ in $\left(\bar{R}^{n}, X\right)$. Since $h_{1}^{-1} D_{1}$ is a compact set in $B_{+}^{k}$, there is a $C$-homeomorphism $f: B^{n} \rightarrow B^{n}$ such that $f B^{k}=B^{k}, f B_{+}^{k} \subset B_{+}^{k} \backslash h_{1}^{-1} D_{1}$, and $f=\mathrm{id}$ near $\partial B^{n}$. Then $h_{1} f h_{1}^{-1}: h_{1} B^{n} \rightarrow h_{1} B^{n}$ can be extended by the identity to a $C$-homeomorphism $g: \bar{R}^{n} \rightarrow \bar{R}^{n}$. Since $g X \subset D_{2}, h_{2}^{-1} g X=Q$ is a $k$-cell in $B_{+}^{k}$. Moreover, $Q$ is locally $C$-flat in $B^{k}$. Since $k-1 \neq 4$, it follows from Theorem 3.3 that $\left(B^{k}, Q\right)$ is $C$-homeomorphic to $\left(B^{k}, \bar{B}^{k}(1 / 2)\right)$. Thus we can find neighbourhoods $U$ and $V$ of $Q$ and $\bar{B}^{k}(1 / 2)$ in $B^{n}$ and a $C$-homeomorphism $\varphi:(U, Q) \rightarrow\left(V, \bar{B}^{k}(1 / 2)\right)$. For $C=\mathrm{LQC}$ and $n \geqq 2$ this requires the extension theorems of [Ah] and [Ca], so $n \leqq 4$. Now $g^{-1} h_{2} \varphi^{-1}: V \rightarrow \bar{R}^{n}$ is a $C$-embedding which maps $\bar{B}^{k}(1 / 2)$ onto $X$. By the Schoenflies theorem, $g^{-1} h_{2} \varphi^{-1} \mid \bar{B}^{k}(1 / 2)$ extends to a $C$-homeomorphism $\left(\bar{R}^{n}, \bar{B}^{k}(1 / 2)\right) \rightarrow$ $\left(\bar{R}^{n}, X\right)$.

(ii) Let $h_{i}: U_{i} \rightarrow \bar{R}^{n}$ be $(C, g)$-encasings of $D_{i}$. Choose a $C$-homeomorphism $u: \bar{R}^{n} \rightarrow \bar{R}^{n}$ so that $u \bar{R}^{k}=\bar{R}^{k}, u E=E_{2}$ and $u=$ id near $\bar{R}^{n} \backslash U_{1}$. Define the $C$-homeomorphism $v: \bar{R}^{n} \rightarrow \bar{R}^{n}$ to be $h_{1} u h_{1}^{-1}$ on $h_{1} U_{1}$ and id elsewhere. Let $U=u^{-1} U_{2}$ and define $f: U \rightarrow \bar{R}^{n}$ by $f=v^{-1} h_{2} u$. Then $f \mid E=g$, and by the Schoenflies theorem there is a self $C$-homeomorphism of $\bar{R}^{n}$ which agrees with $f$ near $E$. 
4.2. Theorem. Suppose that $X \subset \bar{R}^{n}$ is a locally $C$-flat TOP $k$-cell and that $k \neq 4,5$. Then $\left(\bar{R}^{n}, X\right)$ is C-homeomorphic to $\left(\bar{R}^{n}, \bar{B}^{k}\right)$.

Proof. Choose a homeomorphism $f: I^{k} \rightarrow X$. Applying the encasing lemma 2.3 (ii) as in the proof of 3.3, we see that every proper compact subset of $\partial X$ is $C$-encasable in $\left(\bar{R}^{n}, X\right)$. Hence we may choose $r>0$ such that if $P=I^{k-1} \times[1-r, 1]$ and $Q_{0}=\operatorname{cl}\left(\left(I^{k-1} \times[-1,1-r]\right) \backslash I^{k-1}(1-r)\right)$, then $f P$ and $f Q_{0}$ are $C$-encasable in $\left(\bar{R}^{n}, X\right)$. Subdivide the cube $I^{k-1}(1-r)$ into cubes $Q_{1}, \ldots, Q_{s}$ so that for each $j, f Q_{j}$ is $C$-encasable, $F_{j}=Q_{0} \cup \ldots \cup Q_{j}$ is a PL $k$-cell, and $Q_{j+1} \cap F_{j}$ is a PL $(k-1)$-cell. Using inductively Lemma $2.3(\mathrm{i})$, we see that $f F_{s}$ is $C$-encasable in $\left(\bar{R}^{n}, X\right)$. The theorem now follows from Lemma 4.1 (i).

4.3. Theorem. Let $g: I^{k} \rightarrow \bar{R}^{n}$ be a locally $C$-flat embedding. Then $g$ extends to a C-homeomorphism of $\bar{R}^{n}$.

Proof. The proof is similar to the proof of 4.2, except that we use (iii) and (iv) of the encasing lemma 2.3 instead of (i) and (ii), and we use (ii) of Lemma 4.1 instead of (i).

4.4. Theorem. Let $X$ be a locally LIP flat LIP $k$-cell in $\bar{R}^{n}$. Then $\left(\bar{R}^{n}, X\right)$ is lipeomorphic to $\left(\bar{R}^{n}, I^{k}\right)$.

Proof. This follows from 4.3 as 3.6 follows from 3.4. At boundary points, an additional extension by reflection is needed.

\section{References}

[Ah] AhLfors, L. V.: Extension of quasiconformal mappings from two to three dimensions. Proc. Nat. Acad. Sci. U.S.A. 51, 1964, 768-771.

[Bi] Bing, R. H.: Locally tame sets are tame. - Ann. of Math. 59, 1954, 145-158.

[Br] Brakes, W. R.: Quickly unknotting topological spheres. - Proc. Amer. Math. Soc. 72, 1978, 413-416.

[Ca] Carleson, L.: The extension problem for quasiconformal mappings. - Contributions to Analysis, edited by L. V. Ahlfors et al., Academic Press, New York-London, 1974, 39-47.

[Ga] Gauld, D. B.: A technique for extending quasiconformal embeddings. - Proceedings of the Joensuu Colloquium on Complex Analysis (to appear).

[GV] Gauld, D. B., and M. K. Vamanamurthy: Quasiconformal extensions of mappings in $n$-space. - Ann. Acad. Sci. Fenn. Ser. A I 3, 1977, 229-246.

[Ge] Gehring, F. W.: Extension theorems for quasiconformal mappings in $n$-space. - J. Analyse Math. 19, 1967, 149-169.

[Ki] KIRBy, R. C.: Stable homeomorphisms and the annulus conjecture. - Ann. of Math. $89,1969,575-582$.

[LeV 1] Lehto O., and K. I. VirTanen: On the existence of quasiconformal mappings with prescribed complex dilatation. - Ann. Acad. Sci. Fenn. Ser. A I 274, 1960, 1-24.

[LeV 2] Lehto O., and K. I. ViRTANEN: Quasiconformal mappings in the plane. - Springer-Verlag, Berlin-Heidelberg-New York, 1973. 
[LuV] LuUkKainen J., and J. VäISÄLä: Elements of Lipschitz topology. - Ann. Acad. Sci. Fenn. Ser. A I 3, 1977, 85-122.

[Mo 1] Morse, E. E.: Affine structures in 3-manifolds IV. - Ann. of Math. 55, 1952, 215-222.

[Mo 2] MoISE, E. E.: Geometric topology in dimensions 2 and 3. - Springer-Verlag, New YorkHeidelberg-Berlin, 1977.

[NV] Näkki R., and M. K. Vamanamurthy: Quasiconformal disks in 3-space. - Ann. Acad. Sci. Fenn. Ser. A I 535, 1973, 1-10.

[Ri] Rickman, S.: Characterization of quasiconformal arcs. - Ann. Acad. Sci. Fenn. Ser. A I 395, 1966, 1-30.

[Ru] Rushing, T. B.: Topological embeddings. - Academic Press, New York-London, 1973.

[Su] Sullivan, D.: Hyperbolic geometry and homeomorphisms. - Proceedings of the Athens, Georgia, Topology Conference 1977 (to appear).

[Ti] TIENARI, M.: Fortsetzung einer quasikonformen Abbildung über einen Jordanbogen. Ann. Acad. Sci. Fenn. Ser. A I 321, 1962, 1-32.

[Vä] VÄISÄLÄ, J.: Lectures on $n$-dimensional quasiconformal mappings. - Lecture Notes in Mathematics 229, Springer-Verlag, Berlin-Heidelberg-New York, 1971.

University of Auckland

Department of Mathematics

Auckland

New Zealand

Received 24 April 1979

\author{
University of Helsinki \\ Department of Mathematics \\ SF-00100 Helsinki 10 \\ Finland
}

